\title{
On Fuzzy Driven Support for SD-Efficient Portfolio Selection ${ }^{\star}$
}

\author{
Włodzimierz Ogryczak ${ }^{1}$ and Andrzej Romaszkiewicz ${ }^{2}$ \\ ${ }^{1}$ Warsaw University of Technology, Institute of Control \& Computation Engineering, \\ Warsaw, Poland \\ wogrycza@ia.pw.edu.pl \\ ${ }^{2}$ Warsaw School of Economics, Collegium of Economic Analysis, Warsaw, Poland \\ romaszki@bluewin.ch
}

\begin{abstract}
The stochastic dominance (SD) is based on an axiomatic model of risk-averse preferences and therefore, the SD-efficiency is an important property of selected portfolios. As defined with a continuum of criteria representing some measures of failure in achieving several targets, the SD does not provide us with a simple computational recipe. While limiting to a few selected target values one gets a typical multiple criteria optimization model approximating the corresponding SD approach. Although, it is rather difficult to justify a selection of a few target values, this difficulty can be overcome with the effective use of fuzzy target values. While focusing on the first degree SD and extending the target membership functions to some monotonic utility functions we get the multiple criteria model which preserves the consistency with both the first degree and the second degree SD. Further applying the reference point methodology to the multiple criteria model and taking advantages of fuzzy chance specifications we get the method that allows to model interactively the preferences by fuzzy specification of the desired distribution. The model itself guarantees that every generated solution is efficient according to the SD rules.
\end{abstract}

\section{Introduction}

The portfolio optimization problem considered in this paper follows the classical formulation and is based on a single period model of investment. At the beginning of a period, an investor allocates his capital among various securities, thus assigning a nonnegative weight (share of the capital) to each security. During the investment period, a security generates a random rate of return. This results in a change of the capital invested (observed at the end of the period) which is measured by the weighted average of the individual rates of return.

Let $J=\{1,2, \ldots, n\}$ denote a set of securities considered for an investment. For each security $j \in J$, its rate of return is represented by a random variable $R_{j}$ with a given mean $z_{j}=\mathbb{E}\left\{R_{j}\right\}$. Further, let $\mathbf{x}=\left(x_{j}\right)_{j=1,2, \ldots, n}$ denote a vector of

\footnotetext{
* The research was partially supported by the Ministry of Science and Information Society Technologies under grant 3T11C 00527.
} 
decision variables $x_{j}$ expressing the weights defining a portfolio. To represent a portfolio, the weights must satisfy a set of constraints that form a feasible set $\mathcal{P}$. The simplest way of defining a feasible set is by a requirement that the weights must sum to one and short sales are not allowed, i.e. $\sum_{j=1}^{n} x_{j}=1$ and $x_{j} \geq 0$ for $j=1, \ldots, n$. Hereafter, it is assumed that $\mathcal{P}$ is a general LP feasible set given in a canonical form as a system of linear equations with nonnegative variables. Each portfolio $\mathbf{x}$ defines a corresponding random variable $R(\mathbf{x})=\sum_{j=1}^{n} R_{j} x_{j}$ that represents the portfolio rate of return. The mean rate of return for portfolio $\mathbf{x}$ is given as $z(\mathbf{x})=\mathbb{E}\{R(\mathbf{x})\}=\sum_{j=1}^{n} z_{j} x_{j}$. Following the seminal work by Markowitz [6], the portfolio optimization problem is modeled as a mean-risk bicriteria optimization problem where $z(\mathbf{x})$ is maximized and some risk measure $\varrho(\mathbf{x})$ is minimized. In the original Markowitz model [6] the risk is measured by the standard deviation or variance while several other risk measures have been later considered thus creating the entire family of mean-risk (Markowitz-type) models 25$]$.

The Markowitz model is frequently criticized as not consistent with axiomatic models of preferences for choice under risk [13. Models consistent with the preference axioms are based on the relations of stochastic dominance or on expected utility theory 314. In stochastic dominance, uncertain returns (random variables) are compared by pointwise comparison of some performance functions constructed from their distribution functions. The right-continuous cumulative distribution function (cdf): $F_{R(\mathbf{x})}(\eta)=\mathbb{P}\{R(\mathbf{x}) \leq \eta\}$. is used to define the first degree stochastic dominance (FSD). The second function is derived from the cdf as $F_{R(\mathbf{x})}^{(2)}(\eta)=\int_{-\infty}^{\eta} F_{R(\mathbf{x})}(\xi) d \xi$ and it defines the second degree stochastic dominance (SSD). Function $F_{R(\mathbf{x})}^{(2)}$, used to define the SSD relation, can also be presented [10] as $F_{R(\mathbf{x})}^{(2)}(\eta)=\mathbb{E}\left\{(\eta-R(\mathbf{x}))_{+}\right\}$where $(.)_{+}$denotes the nonnegative part. Hence, while function $F_{R(\mathbf{x})}$ expresses the probability of a shortfall to target $\eta$, function $F_{R(\mathbf{x})}^{(2)}$ measures the mean shortfall to the target. The weak relations of stochastic dominance (FSD or SSD) are defined by pointwise inequalities for all real targets: $R\left(\mathbf{x}^{\prime}\right) \succeq_{F S D} R\left(\mathbf{x}^{\prime \prime}\right)$ if $F_{R\left(\mathbf{x}^{\prime}\right)}(\eta) \leq F_{R\left(\mathbf{x}^{\prime \prime}\right)}(\eta)$ for all $\eta$, and respectively, $R\left(\mathbf{x}^{\prime}\right) \succeq_{S S D} R\left(\mathbf{x}^{\prime \prime}\right)$ if $F_{R\left(\mathbf{x}^{\prime}\right)}^{(2)}(\eta) \leq F_{R\left(\mathbf{x}^{\prime \prime}\right)}^{(2)}(\eta)$ for all $\eta$. We say that portfolio $\mathbf{x}^{\prime}$ dominates $\mathbf{x}^{\prime \prime}$ under the FSD (SSD) if $F_{R\left(\mathbf{x}^{\prime}\right)}^{(1)}(\eta) \leq F_{R\left(\mathbf{x}^{\prime \prime}\right)}^{(1)}(\eta)$ $\left(F_{R\left(\mathbf{x}^{\prime}\right.}^{(2)}\right)(\eta) \leq F_{R\left(\mathbf{x}^{\prime \prime}\right)}^{(2)}(\eta)$, respectively) for all $\eta$, with at least one strict inequality. A feasible portfolio $\mathbf{x}^{0} \in \mathcal{P}$ is called FSD (SSD) efficient if there is no $\mathbf{x} \in \mathcal{P}$ such that $R(\mathbf{x}) \succ_{F S D} R\left(\mathbf{x}^{0}\right)\left(R(\mathbf{x}) \succ_{S S D} R\left(\mathbf{x}^{0}\right)\right)$.

We consider $T$ scenarios with probabilities $p_{t}$ (where $t=1, \ldots, T$ ). We assume that for each random variable $R_{j}$ its realization $r_{j t}$ under the scenario $t$ is known. Typically, the realizations are derived from historical data treating $T$ historical periods as equally probable scenarios $\left(p_{t}=1 / T\right)$. The realizations of the portfolio return $R(\mathbf{x})$ are given as $r_{t}(\mathbf{x})=\sum_{j=1}^{n} r_{j t} x_{j}$ and the expected value can be computed as $z(\mathbf{x})=\sum_{t=1}^{T} r_{t}(\mathbf{x}) p_{t}=\sum_{t=1}^{T}\left[\sum_{j=1}^{n} r_{j t} x_{j}\right] p_{t}$. Similarly, values of functions $F_{R(x)}^{(1)}(\eta)$ and $F_{R(x)}^{(2)}(\eta)$ can easily be computed then for any given 
target $\eta$. Nevertheless, as defined with a continuum of criteria representing some measures of failure in achieving several targets, the stochastic dominance models do not provide us with a simple computational recipe. While limiting to a few selected target values one gets typical multiple criteria optimization models approximating the corresponding stochastic dominance approaches $7,8,9]$. However, in practice, it is rather difficult to justify a selection of a few target values. This difficulty can be overcome with the effective use of fuzzy target values.

\section{Fuzzy Targets}

Let us focus on the FSD model. Although the right-continuous cdf defines the FSD relation, the left-continuous cdf $F_{R(\mathbf{x})}^{(1)}(\eta)=\mathbb{P}\{R(\mathbf{x})<\eta\}$ can equivalently be used for this purpose. It provides a lucid interpretation of the FSD relation as pointwise comparison of downside (below-target) risk measures representing probabilities of not achieving given levels (targets), as well as it allows us to build a simple optimization model for those measures. Suppose one has preselected $m$ return values $\eta_{1}>\eta_{2}>\ldots>\eta_{m}$ as targets to evaluate probabilities of the corresponding shortfalls. Introducing $m$ corresponding criteria

$$
s_{k}(\mathbf{x})=F_{R(\mathbf{x})}^{(1)}\left(\eta_{k}\right) \quad \text { for } k=1,2, \ldots, m
$$

one gets the multiple criteria portfolio optimization model:

$$
\min \left\{\left(s_{1}(\mathbf{x}), s_{2}(\mathbf{x}), \ldots, s_{m}(\mathbf{x})\right): \mathbf{x} \in \mathcal{P}\right\}
$$

Due to the use of the left-continuous cdf as criteria $s_{k}(\mathbf{x})=F_{R(\mathbf{x})}^{(1)}\left(\eta_{k}\right)$, optimization (2) can be formulated as mixed integer linear programming problem

$$
\begin{aligned}
& \min \left(y_{1}, y_{2}, \ldots, y_{m}\right) \quad \text { s.t. } \mathbf{x} \in \mathcal{P}, \quad y_{k}=\sum_{t=1}^{T} b_{k t} p_{t} \quad \text { for } k=1, \ldots, m \\
& M b_{k t} \geq \eta_{k}-\sum_{j=1}^{n} r_{j t} x_{j}, \quad b_{k t} \in\{0,1\} \quad \text { for } k=1, \ldots, m ; t=1, \ldots, T
\end{aligned}
$$

where $M$ a sufficiently large constant and $b_{k t}$ are binary variables taking value 1 whenever realization of the portfolio return under scenario $t$ is below the target value $\eta_{k}$ (i.e., $\left.b_{k t} \geq \operatorname{sign}\left(\left(\eta_{k}-r_{t}(\mathbf{x})\right)_{+}\right)\right)$.

Model (2) represents an approximation to the FSD approach. One can easily notice that any portfolio $\overline{\mathbf{x}} \in \mathcal{P}$ efficient (Pareto-optimal) solution to (2) can be FSD dominated only by an alternative efficient portfolio $\mathbf{x} \in \mathcal{P}$ with the same values of criteria $s_{k}(\mathbf{x})=s_{k}(\overline{\mathbf{x}})$ for all $k=1, \ldots, m$. Hence, a small number of targets results in serious threat of FSD ambiguity in the sense the selected portfolio efficient to the multiple criteria model can easily be FSD dominated by another quite a different portfolio with the same values of the cdf at the few targets. 
Note that values $\eta_{k}$ actually represent the targets defined as corresponding (closed) crisp sets $\left\{\eta \in \mathbb{R} \mid \eta \geq \eta_{k}\right\}$, and their characteristic functions

$$
\chi_{k}(\eta)=\left\{\begin{array}{l}
0 \text { for } \eta<\eta_{k} \\
1 \text { for } \eta \geq \eta_{k}
\end{array}\right.
$$

allow to express the FSD criteria as $s_{k}(\mathbf{x})=\mathbb{E}\left\{1-\chi_{k}(R(\mathbf{x}))\right\}$. Taking advantages of the discrete distribution we have assumed they can be written as $s_{k}(\mathbf{x})=$ $1-\sum_{t=1}^{T} \chi_{k}\left(r_{t}(\mathbf{x})\right) p_{t}$. We propose to replace crisp targets with fuzzy targets $C_{k}$. We will focus on trapezoidal fuzzy sets defined by nondecreasing piecewise linear membership functions:

$$
\mu_{k}(\eta)= \begin{cases}0 & \text { for } \eta<\eta_{k}^{-} \\ \left(\eta-\eta_{k}^{-}\right) /\left(\eta_{k}^{+}-\eta_{k}^{-}\right) & \text {for } \eta_{k}^{-} \leq \eta<\eta_{k}^{+} \\ 1 & \text { for } \eta \geq \eta_{k}^{+}\end{cases}
$$

defined by two parameters (breakpoints) $\eta_{k}^{-}$and $\eta_{k}^{+}$representing the largest return with membership level 0 and the smallest return with membership level 1 , respectively. To relate them with values $\eta_{k}$ defining the crisp targets we will assume that $\eta_{k}^{+}=\eta_{k}$ while $\eta_{k}^{-}=\eta_{k}-\Delta_{k}$ for a given fuzzyfication parameter $\Delta_{k}>0$. Hence, the fuzzy targets $C_{k}$ can be specified by the interval $\left[\eta_{k}^{-}, \eta_{k}^{+}\right]$or equivalently by the pair of numbers $\left(\eta_{k}, \Delta_{k}\right)$.

With fuzzy targets $C_{k}$, the corresponding FSD criteria are expressed as

$$
s_{k}(\mathbf{x})=\mathbb{E}\left\{1-\mu_{k}(R(\mathbf{x}))\right\}=\frac{1}{\Delta_{k}} \int_{\eta_{k}-\Delta_{k}}^{\eta_{k}} F_{R(\mathbf{x})}(\eta) d \eta
$$

Applying them in multiple criteria model (2) one gets the fuzzy portfolio optimization model which offers more intuitive way to define targets. It provides an opportunity to define expectations in fuzzy terms like: "minimize the probability of shortfall to medium profit." Moreover, due to aggregation on intervals $\left[\eta_{k}-\Delta_{k}, \eta_{k}\right]$ made in (4), the following assertion is valid.

Proposition 1. Except for portfolios with identical cdf values within all intervals $\left[\eta_{k}-\Delta_{k}, \eta_{k}\right]$, every portfolio $\mathbf{x} \in \mathcal{P}$ efficient to the multiple criteria problem (2) with fuzzy defined criteria (4) is FSD efficient.

Proof. Let $\overline{\mathbf{x}} \in \mathcal{P}$ be an efficient solution to the multiple criteria problem (2) with criteria (4). IF it is FSD dominated by portfolio $\mathbf{x} \in \mathcal{P}$, then $F_{R(\mathbf{x})}(\eta) \leq F_{R(\overline{\mathbf{x}})}(\eta)$ for all $\eta$ with at least one inequality strict. Hence, due to (4), $s_{k}(\overline{\mathbf{x}}) \geq s_{k}(\mathbf{x})$ for all $k$ and any strict inequality $F_{R(\mathbf{x})}(\eta)<F_{R(\overline{\mathbf{x}})}(\eta)$ for some $\eta \in\left[\eta_{k}-\Delta_{k}, \eta_{k}\right]$ would result in strict inequality $s_{k}(\overline{\mathbf{x}})>s_{k}(\mathbf{x})$ thus contradicting the efficiency of $\overline{\mathbf{x}}$ to (2).

Note that distributions of rates of return are usually characterized by bounded support which can easily be covered by the interval $\left[\eta_{m}-\Delta_{m}, \eta_{1}\right]$ while all $\Delta_{k} \geq \eta_{k}-\eta_{k+1}$. Following Proposition 1, every portfolio efficient to the corresponding multiple criteria problem (2) with fuzzy defined criteria (4) is then (unconditionally) FSD efficient. 
Problem (2) with fuzzy defined criteria (41) can be expressed as

$$
\min \left\{\left(1-\mathbb{E}\left\{\mu_{1}(R(\mathbf{x}))\right\}, 1-\mathbb{E}\left\{\mu_{2}(R(\mathbf{x}))\right\}, \ldots, 1-\mathbb{E}\left\{\mu_{m}(R(\mathbf{x}))\right\}\right): \mathbf{x} \in \mathcal{P}\right\}
$$

Hence, taking advantages of the discrete distributions, it can be formulated as the following mixed integer programming problem:

$$
\begin{gathered}
\min \left(y_{1}, y_{2}, \ldots, y_{m}\right) \quad \text { s.t. } \mathbf{x} \in \mathcal{P}, \quad y_{k}=\sum_{t=1}^{T} v_{k t} p_{t} \quad \text { for } k=1, \ldots, m \\
v_{k t} \geq b_{k t}, \quad b_{k t} \in\{0,1\} \quad \text { for } k=1, \ldots, m ; t=1, \ldots, T \\
\Delta_{k} v_{k t}+M b_{k t} \geq \eta_{k}-\sum_{j=1}^{n} r_{j t} x_{j} \quad \text { for } k=1, \ldots, m ; t=1, \ldots, T
\end{gathered}
$$

where $M$ a sufficiently large constant and $b_{k t}$ are binary variables taking value 1 whenever realization of the portfolio return under scenario $t$ is below the value $\eta_{k}-\Delta_{k}$ while $v_{k t} \geq 1-\mu_{k}\left(r_{t}(\mathbf{x})\right)$.

\section{Extension to Concave Utility}

Membership functions $\mu_{k}$ in criteria $1-\mathbb{E}\left\{\mu_{k}(R(\mathbf{x}))\right\}$ of problem (5) may be interpreted as utility functions used to maximize the corresponding expected utility value. In order to guarantee full consistency of the expected utility maximization with FSD the utility function must be strictly increasing. Moreover concave utility function represent risk aversion (SSD consistency). Membership function $\mu_{k}$ is strictly increasing within the interval $\left[\eta_{k}^{-}, \eta_{k}^{+}\right]$while being constant for $\eta$ smaller than $\eta_{k}^{-}$or larger than $\eta_{k}^{+}$. Moreover, it is neither concave nor convex. Nevertheless, one can easily extend $\mu_{k}$ to a strictly increasing concave utility function $\bar{\mu}_{k}$ preserving the original values on interval $\left[\eta_{k}^{-}, \eta_{k}^{+}\right]$. The simplest such an extension is given by the following piecewise linear function:

$$
\bar{\mu}_{k}(\eta)= \begin{cases}\left(\eta-\eta_{k}^{-}\right) /\left(\eta_{k}^{+}-\eta_{k}^{-}\right) & \text {for } \eta<\eta_{k}^{+} \\ 1+\beta\left(\eta-\eta_{k}^{+}\right) /\left(\eta_{k}^{+}-\eta_{k}^{-}\right) & \text {for } \eta \geq \eta_{k}^{+}\end{cases}
$$

where $0<\beta<1$ is an arbitrary small positive constant (Fig. 1). Note that function (7) can alternatively be expressed in terms of $\eta_{k}^{+}=\eta_{k}$ and $\eta_{k}^{-}=\eta_{k}-\Delta_{k}$ as

$$
\bar{\mu}_{k}(\eta)=1-\beta\left(\eta_{k}-\eta\right) / \Delta_{k}-(1-\beta)\left(\eta_{k}-\eta\right)_{+} / \Delta_{k}
$$

Reformulating (5) with functions $\bar{\mu}_{k}$ we get the multiple criteria problem

$$
\min \left\{\left(1-\mathbb{E}\left\{\bar{\mu}_{1}(R(\mathbf{x}))\right\}, 1-\mathbb{E}\left\{\bar{\mu}_{2}(R(\mathbf{x}))\right\}, \ldots, 1-\mathbb{E}\left\{\bar{\mu}_{m}(R(\mathbf{x}))\right\}\right): \mathbf{x} \in \mathcal{P}\right\}
$$

Since $\bar{\mu}_{k}$ are no longer fuzzy membership functions, the problem is not a fuzzy optimization model. It is a fuzzy driven multiple criteria model as the utility functions are defined by parameters of fuzzy targets. However, the use of utility 

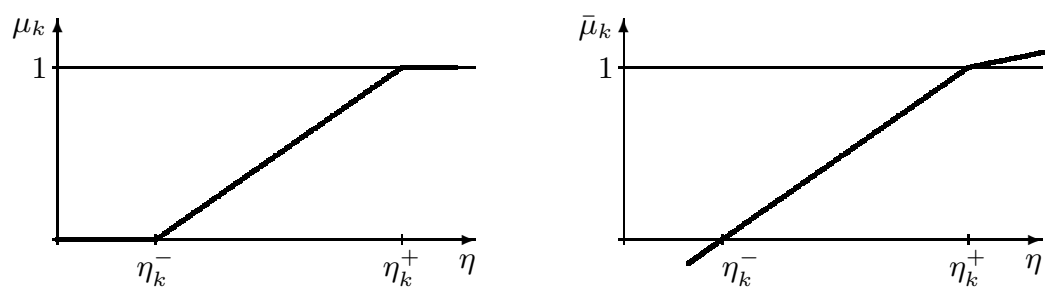

Fig. 1. Membership functions $\mu_{k}$ and increasing concave utility function $\bar{\mu}_{k}$

functions in (9) guarantees the model consistency with the SD rules. Note that, due to (8)

$$
\begin{aligned}
1-\mathbb{E}\left\{\bar{\mu}_{k}(R(\mathbf{x}))\right\} & =\frac{\beta}{\Delta_{k}}\left(\eta_{k}-\mathbb{E}\{R(\mathbf{x})\}\right)+\frac{(1-\beta)}{\Delta_{k}} \int_{-\infty}^{\eta_{k}} F_{R(\mathbf{x})}(\eta) d \eta \\
& =\left[\beta\left(\eta_{k}-z(\mathbf{x})\right)+(1-\beta) F_{R(\mathbf{x})}^{(2)}\left(\eta_{k}\right)\right] / \Delta_{k}
\end{aligned}
$$

thus allowing us to prove the following consistency statements.

Proposition 2. Every portfolio $\mathrm{x} \in \mathcal{P}$ efficient to multiple criteria problem (9) is FSD efficient.

Proof. Suppose that $\overline{\mathbf{x}} \in \mathcal{P}$ efficient to (9) is FSD dominated by portfolio $\mathbf{x} \in \mathcal{P}$, i.e. $F_{R(\mathbf{x})}(\eta) \leq F_{R(\overline{\mathbf{x}})}(\eta)$ for all $\eta$ with at least one inequality strict. Then $z(\mathbf{x})>$ $z(\overline{\mathbf{x}})$ and $F_{R(\mathbf{x})}^{(2)}\left(\eta_{k}\right) \leq F_{R(\overline{\mathbf{x}})}^{(2)}\left(\eta_{k}\right)$ for all $k$. Hence. due to $(10), 1-\mathbb{E}\left\{\bar{\mu}_{k}(R(\overline{\mathbf{x}}))\right\}>$ $1-\mathbb{E}\left\{\bar{\mu}_{k}(R(\mathbf{x}))\right\}$ for all $k$ thus contradicting the efficiency of $\overline{\mathbf{x}}$ to (9).

Proposition 3. Except for portfolios with identical values of the expected utility criteria $\mathbb{E}\left\{\bar{\mu}_{k}(R(\mathbf{x}))\right\}$, every portfolio $\mathbf{x} \in \mathcal{P}$ efficient to the multiple criteria problem (9) is SSD efficient.

Proof. If $\overline{\mathbf{x}} \in \mathcal{P}$ is SSD dominated by $\mathbf{x} \in \mathcal{P}$, then $F_{R(\mathbf{x})}^{(2)}(\eta) \leq F_{R(\overline{\mathbf{x}})}^{(2)}(\eta)$ for all $\eta$ and $z(\mathbf{x}) \geq z(\overline{\mathbf{x}})[10$. Hence. due to (10) $), 1-\mathbb{E}\left\{\bar{\mu}_{k}(R(\overline{\mathbf{x}}))\right\}>1-\mathbb{E}\left\{\bar{\mu}_{k}(R(\mathbf{x}))\right\}$ for all $k$ and actually $1-\mathbb{E}\left\{\bar{\mu}_{k}(R(\overline{\mathbf{x}}))\right\}=1-\mathbb{E}\left\{\bar{\mu}_{k}(R(\mathbf{x}))\right\}$ for all $k$ due to the efficiency of $\overline{\mathbf{x}}$ to (9).

Taking advantages of the discrete distributions, problem (9) can be formulated as the following linear programming problem. Note that concave piecewise linear utility functions replacing the fuzzy membership functions allow us to eliminate binary variables form the optimization problem thus leading to the following linear programming model

$$
\begin{gathered}
\min \left(y_{1}, y_{2}, \ldots, y_{m}\right) \quad \text { s.t. } \mathbf{x} \in \mathcal{P}, \quad y_{k}=\sum_{t=1}^{T} v_{k t} p_{t} \quad \text { for } k=1, \ldots, m \\
\Delta_{k} v_{k t} \geq \beta\left(\eta_{k}-\sum_{j=1}^{n} r_{j t} x_{j}\right) \quad \text { for } k=1, \ldots, m ; t=1, \ldots, T \\
\Delta_{k} v_{k t} \geq \eta_{k}-\sum_{j=1}^{n} r_{j t} x_{j} \quad \text { for } k=1, \ldots, m ; t=1, \ldots, T
\end{gathered}
$$




\section{Reference Point Method and Fuzzy Chances}

An operational use of multiple criteria model (9) requires to select one efficient portfolio for implementation. This can be achieved with the so-called quasisatisficing approach to multiple criteria optimization introduced as the reference point method [15] and later extended the aspiration/reservation based decision support (ARBDS) approach with many successful applications [4]. In the ARBDS interactive scheme the decision maker (DM) specifies requirements in terms of aspiration and reservation levels, i.e., by introducing acceptable and required values for several criteria. Depending on the specified aspiration and reservation levels, a special scalarizing achievement function is built which generates an efficient solution to the multiple criteria problem when maximized. The computed efficient solution is presented to the DM as the current solution in a form that allows comparison with the previous ones and modification of the aspiration and reservation levels if necessary.

The scalarizing achievement function must be strictly monotonic with respect to each outcome (decreasing for the minimization problem (91)). Second, a solution with all individual outcomes $y_{k}$ satisfying the corresponding reservation levels is preferred to any solution with at least one individual outcome worse than its reservation level. Next, provided that all the reservation levels are satisfied, a solution with all individual outcomes $y_{k}$ equal to the corresponding aspiration levels is preferred to any solution with at least one outcome worse than its aspiration level. The generic scalarizing achievement function takes the following form [15]:

$$
a(\mathbf{y})=\min _{1 \leq k \leq m}\left\{a_{k}\left(y_{k}\right)\right\}+\varepsilon \sum_{i=k}^{m} a_{k}\left(y_{k}\right)
$$

where $\varepsilon$ is an arbitrary small positive number and $a_{k}$ are the partial achievement functions measuring actual achievement of outcome $y_{k}$ with respect to the corresponding aspiration and reservation levels $\left(y_{k}^{a}<y_{k}^{r}\right.$, respectively). Thus the scalarizing achievement function is, essentially, defined by the worst partial achievement but additionally regularized with the sum of all partial achievements. The regularization term is introduced only to guarantee the solution efficiency in the case when the maximization of the main term (the worst partial achievement) results in a non-unique optimal solution.

The partial achievement function $a_{k}$ can be interpreted as a measure of the DM's satisfaction with the current value of outcome of the $k$ th criterion. It is a strictly decreasing function of outcome $y_{k}$ with value $a_{k}=1$ if $y_{k}=y_{k}^{a}$, and $a_{k}=0$ for $y_{i}=y_{k}^{r}$. Various functions can be built meeting those requirements. We use the piecewise linear partial achievement function given by

$$
a_{k}\left(y_{k}\right)= \begin{cases}\left(y_{k}^{r}-y_{k}\right) /\left(y_{k}^{r}-y_{k}^{a}\right) & \text { for } y_{k}>y_{k}^{a} \\ 1+\alpha\left(y_{k}^{a}-y_{k}\right) /\left(y_{k}^{r}-y_{k}^{a}\right) & \text { for } y_{k} \leq y_{k}^{a}\end{cases}
$$

where $\alpha$ is arbitrarily defined small parameter satisfying $0<\alpha<1$ representing additional increase of the DM's satisfaction over level 1 when a criterion 
generates outcomes better than the corresponding aspiration level. Partial achievement function (13) is strictly decreasing and concave piecewise linear function, which guarantees its LP computability with respect to outcomes $y_{k}$. Finally, maximization of the entire scalarizing achievement function (12) can be implemented by auxiliary LP constraints thus preserving the LP structure of the entire RPM model:

$$
\max \left\{a(\mathbf{y}): \mathbf{x} \in \mathcal{P}, \quad y_{k}=1-\mathbb{E}\left\{\bar{\mu}_{k}(R(\mathbf{x}))\right\} \quad \text { for } k=1, \ldots, m\right\}
$$

Model (14) generates efficient solutions to the corresponding problem (9). Hence, due to Propositions 2 and 3 , the following assertions are valid.

Proposition 4. Every portfolio $\mathbf{x} \in \mathcal{P}$ optimal to the RPM problem (14) is FSD efficient.

Proposition 5. Except for portfolios with identical values of the expected utility criteria $\mathbb{E}\left\{\bar{\mu}_{k}(R(\mathbf{x}))\right\}$, every portfolio $\mathbf{x} \in \mathcal{P}$ optimal to the RPM problem (14) is SSD efficient.

For outcomes between the reservation and the aspiration levels, the partial achievement function $a_{k}$ can be interpreted as a trapezoidal membership function $\mu_{k}$ for a fuzzy generalization of the crisp target $\left\{y_{k}: y_{k} \leq y_{k}^{a}\right\}$. Hence, the partial achievement function (13), similar to (7), can be viewed as an extension of the fuzzy membership function to a strictly monotonic and concave utility. One may also notice that the aggregation scheme used to build the scalarizing achievement function (12) from the partial ones may be interpreted as some fuzzy aggregation operator using the ordered weighted averaging 16. In other words, maximization of the scalarizing achievement function (12) is consistent with the fuzzy optimization methodology and the aspiration and reservation levels may be regarded as the specification of fuzzy targets for the criteria values. Actually, as the original criteria $s_{k}$ represent the shortfall probabilities, the aspiration and reservation levels represent the fuzzy chances. For instance, to seek low chance of losses and high chance of medium profit one may specify two (criteria) fuzzy targets: $\left(\eta_{1}^{-}, \eta_{1}^{+}\right)=(0.00,0.02)$ with $y_{1}^{a}=0.0$ and $y_{1}^{r}=0.02$ and $\left(\eta_{2}^{-}, \eta_{2}^{+}\right)=(0.02,0.05)$ with $y_{2}^{a}=0.2$ and $y_{2}^{r}=0.5$. Sample list of such simple fuzzy terms for portfolio selection is given in Table 1 .

In our initial tests we have applied the model to the portfolio selection within the set of assets representing 32 major stock exchange indexes from various countries 1 The analysis was based on the period 04.07.1997-24.09.2004 with the quotations every 4 weeks. The tests [12] has confirmed easiness of the interactive preference specification with fuzzy targets and fuzzy chances as well as good quality of generated portfolios with respect to the classical Markowitz criteria. Here we present an illustrative example of a simple analysis performed using only simplified fuzzy terms from Table 1 .

We start with the requirement to find a portfolio with low chance of losses. This requirement leads us to the single-criterion model $(m=1)$ defined by

${ }^{1}$ The quotation data supplied by http://finance.yahoo.com 
Table 1. Sample list of fuzzy terms

\begin{tabular}{l|c|c}
\hline Fuzzy targets & Definition & $\left(\eta^{-}, \eta^{+}\right)$ \\
\hline losses & rate of return below 2\% & $(0.00,0.02)$ \\
medium profit & rate of return about 5\% & $(0.02,0.05)$ \\
large profit & rate of return about 10\% & $(0.05,0.10)$ \\
\hline Fuzzy chances & Definition & $\left(y^{r}, y^{a}\right)$ \\
\hline low chance & prob. below 2\% & $(0.00,0.02)$ \\
medium chance & $\begin{array}{l}\text { prob. about 50\% } \\
\text { prob. about 80\% }\end{array}$ & $(0.20,0.50)$ \\
high chance & $(0.50,0.80)$ \\
\hline
\end{tabular}

Table 2. Sample portfolios characteristics

\begin{tabular}{l|llll}
\hline & $\mathrm{P} 1$ & $\mathrm{P} 2$ & $\mathrm{P} 3$ & $\mathrm{P} 4$ \\
\hline Expected return rate & 1.011 & 1.015 & 1.02 & 1.013 \\
Variance & 0.0008 & 0.0015 & 0.0044 & 0.0011 \\
Mean absolute deviation & 0.0284 & 0.029 & 0.0472 & 0.0254 \\
\hline
\end{tabular}

the fuzzy target $\left(\eta^{-}, \eta^{+}\right)=(0.00,0.02)$ and the aspiration/reservation pair $\left(y^{r}, y^{a}\right)=(0.00,0.02)$. Note that, due to the use of model (14) with strictly monotonic utility function $\bar{\mu}$ all probabilities of underachievements are minimized despite only losses are specified as a fuzzy target. This results in a FSD efficient portfolio (Proposition 4) presented as portfolio P1 in Table 2 where simplified portfolio characteristics are reported. Next, we try to examine a possibility of achieving portfolios with higher returns by maximizing the chance of getting medium or high return, respectively. This leads us to the singlecriterion models $(m=1)$ defined by the fuzzy targets $\left(\eta^{-}, \eta^{+}\right)=(0.02,0.05)$ and $\left(\eta^{-}, \eta^{+}\right)=(0.05,0.10)$, respectively. In both models the aspiration/reservation pair $\left(y^{r}, y^{a}\right)=(0.50,0.80)$ is used to represent high chance of reaching the results. The corresponding portfolios are presented as P2 and P3 in Table2 Again, following Proposition 4, both the portfolios are FSD efficient. When comparing to $\mathrm{P} 1$, portfolio $\mathrm{P} 2$ (high chance of medium return) is characterized by more than $25 \%$ increase of the expected return while preserving very similar level risk measured with mean absolute deviation (mean absolute deviation is a risk measure consistent with the FSD and SSD orders whereas variance not [8]). Portfolio P3 (high chance of high return) reaches almost doubled expected return but with similar increase of the mean absolute deviation. Finally, in order to find a portfolio with low chance of losses and high chance to achieve medium return we solve a bicriteria model $(m=2)$ defined by the fuzzy targets $\left(\eta_{1}^{-}, \eta_{1}^{+}\right)=(0.00,0.02)$ and $\left(\eta_{2}^{-}, \eta_{2}^{+}\right)=(0.02,0.05)$. Generated FSD efficient portfolio P4 is indeed characterized by medium return and low risk (mean absolute deviation). Thus, it might be accepted as the final solution. 


\section{Concluding Remarks}

Application of the FSD criteria to a few fuzzy targets of rate of return with membership functions expanded to monotonic concave utility functions allows us to specify easily an FSD and SSD consistent multiple criteria portfolio optimization model. Further, formally FSD criteria allow us to define aspiration and reservation levels via fuzzy definition of chances. The resulting RPM model is SSD consistent while allowing for easily defined preference parameters controlling the interactive analysis. The complete model is LP computable in the case of discrete random variables (historical data). The initial tests [12] has confirmed easiness of the interactive preference specification with fuzzy targets and fuzzy chances as well as good quality of generated portfolios with respect to the classical Markowitz criteria.

\section{References}

1. Bawa, V.S.: Safety-first, stochastic dominance and optimal portfolio choice. J. Finan. Quant. Anal. 13 (1978) 252-271.

2. Krzemienowski, A., Ogryczak, W.: On extending the LP computable risk measures to account downside risk. Comput. Optimization and Appls., 32, (2005), 133-160.

3. Levy, H.: Stochastic dominance and expected utility: survey and analysis. Manage. Sci. 38 (1992) 555-593.

4. Lewandowski, A., Wierzbicki, A.P. (eds.): Aspiration Based Decision Support Systems - Theory, Software and Applications. Springer, Berlin, 1989.

5. Mansini, R., Ogryczak, W., Speranza, M.G.: On LP solvable models for portfolio selection. Informatica, 14, (2003), 37-62.

6. Markowitz, H.M.: Portfolio selection. J. Fin. 7 (1952) 77-91.

7. McNamara, J.R.: Portfolio selection using stochastic dominance criteria. Decision Sci, 29 (1998) 785-801.

8. Ogryczak, W.: Multiple criteria linear programming model for portfolio selection. Ann. Oper. Res. 97 (2000) 143-162.

9. Ogryczak, W.: Multiple criteria optimization and decisions under risk. Control \& Cybernetics, 31, (2002), 975-1003.

10. Ogryczak, W., Ruszczyński, A.: From stochastic dominance to mean-risk models: semideviations as risk measures. Eur. J. Oper. Res. 116 (1999) 33-50.

11. Ribeiro, R.A., Zimmermann, H.-J., Yager, R.R., Kacprzyk, J. (eds.): Soft Computing in Financial Engineering, Physica-Verlag, Heidelberg, 1999.

12. Romaszkiewicz, A., Portfolio selection with techniques of fuzzy and multicriteria optimization (in Polish). Ph.D. Thesis, Warsaw School of Economics, 2006.

13. Rothschild, M., Stiglitz, J.E.: Increasing risk: I. A definition. J. Econ. Theory 2 (1969) 225-243.

14. Whitmore, G.A., Findlay, M.C. (eds.): Stochastic Dominance: An Approach to Decision-Making Under Risk, D.C.Heath, Lexington MA 1978.

15. Wierzbicki, A.P.: A mathematical basis for satisficing decision making. Math. Modelling 3 (1982) 391-405.

16. Yager, R.R., Filev, D.P.: Essentials of Fuzzy Modelling and Control, Wiley, NY, 1994. 